\title{
Plasma miR-15b-5p and miR-590-5p for distinguishing patients with bladder cancer from healthy individuals
}

\author{
ANGELIKA TÖLLE ${ }^{1,2}$, LAURA BUCKENDAHL ${ }^{1}$ and KLAUS JUNG ${ }^{1,3}$ \\ ${ }^{1}$ Department of Urology, Charité-Universitätsmedizin Berlin, D-10117 Berlin; \\ ${ }^{2}$ CONGEN Biotechnology GmbH, D-13125 Berlin; ${ }^{3}$ Berlin Institute for Urologic Research, D-10117 Berlin, Germany
}

Received February 12, 2019; Accepted June 5, 2019

DOI: 10.3892/or.2019.7247

\begin{abstract}
MicroRNAs (miRNAs/miRs) act as markers for various cancers, including bladder cancer (BC). Plasma miRNAs are potential biomarkers for the noninvasive diagnosis and long-term surveillance of BC. The aim of the present study was to identify diagnostically reliable miRNAs in the plasma and examine their potential monitoring capacity. miRNAs were measured by reverse transcription-quantitative polymerase chain reaction. The study was performed as a discovery phase, which included plasma samples from each of the 10 patients with and without $\mathrm{BC}$ prior to transurethral resection (TURB). The results were validated in a second phase, involving 36 patients with plasma samples collected before the second TURB or radical cystectomy (RC), and after RC. During the discovery step, three elevated miRNAs (miR-15b-5p, miR-590-5p, miR-29b-3p) and two decreased miRNAs (miR-10b-5p, miR-144-5p) were selected as potential miRNA candidates for further validation. miR-15b-5p and miR-590-5p were finally confirmed to discriminate between cancer cases and controls; however, for disease monitoring of BC, both miRNAs were not suitable as a decline in the miRNA levels was not observed in some patients after tumor removal. Our results suggested that circulating miR-15b-5p and miR-590-5p have useful diagnostic potential for $\mathrm{BC}$, but are rather unsuitable as monitoring markers for disease. The reasons of this apparent contradictory observation may be due to the aspect of biological variation of circulating miRNAs and serial measurements could be unreliable.
\end{abstract}

Correspondence to: Dr Angelika Tölle, Department of Urology, Charité-Universitätsmedizin Berlin, Charitèplatz 1, D-10117 Berlin, Germany

E-mail: angelika.toelle@charite.de

Abbreviations: AUC, area under the curve; BC, bladder cancer; CI, confidence interval; $\mathrm{Cq}$, quantification cycle; miRNAs, microRNAs; $\mathrm{RC}$, radical cystectomy; RT-qPCR, reverse transcription-quantitative polymerase chain reaction; TURB, transurethral resection; YAP, Yes associated protein

Key words: bladder cancer, diagnosis, miRNA, plasma, monitoring

\section{Introduction}

Urinary bladder cancer (BC) is the most common tumor of the urinary system. In the United States of America alone, 79,030 new cases and 16,870 mortalities were registered in 2017 due to urinary BC (1). These account for $50 \%$ of all tumors of the urinary system (1). Cystoscopy remains as the diagnostic 'gold standard' for primary diagnosis and follow-up of bladder cancer. At the point of diagnosis, the majority of BC cases $(75 \%)$ are limited to the organ and are characterized as non-muscle invasive bladder tumors (NMIBC) which affect only the mucosa or submucosa (2). The remaining tumors are muscle-invasive tumors (MIBC) which spread to the deeper layers of tissue. For NMIBC, alterations in the fibroblast growth factor $(F G F)$ receptor 3 gene are common (3), while for MIBC, mutations in P53, Rbl and phosphatase and tensin homolog have been reported (4). After initial diagnosis and treatment, recurrence is likely to occur; thus, patients must undergo a long period of surveillance (2). Repeated cystoscopies are uncomfortable for patients, time consuming for physicians and expensive. The main objectives of urological research are to improve early diagnosis and the surveillance of patients (5), and reduce the subsequent cystoscopies by analyzing biomarkers in body fluids. Furthermore, the identification of novel markers of therapeutic responses is required to reduce the number of necessary transurethral resection of bladder tumor (TURB) procedures for patients with BC. In this respect, the altered expression of microRNAs (miRNAs/miRs) in the body fluids of patients, including blood and urine, is of considerable interest. A systematic review reported this for such patients and those with other urological cancers (6). The advantage of miRNAs is their stability in body fluids and the possibility of non-invasive collection of samples (7).

Six studies have investigated differentially expressed miRNAs in the plasma of BC patients; the findings suggested that circulating miRNAs in the plasma could be novel non-invasive biomarkers for bladder cancer (8-13). However, the focus of these studies was primarily the diagnostic validity of miRNAs. The potential monitoring capacity during the surveillance of these patients has not yet been studied. In addition, inconsistent results have been reported from such studies, not only from the heterogeneity of study cohorts but also due to analytical differences in the sampling processes and different normalization approaches (14). Considering 
these critical points, the objectives of the present study were: i) To examine a broad panel of plasma miRNAs and identify potential diagnostic and surveillance monitoring markers and useful reference miRNAs; and ii) to focus on the pre-analytical process to exclude samples with interfering analytical factors, such as the hemolysis of plasma samples. Based on the promising results of previous studies using plasma (8-13), we also decided to determine miRNAs in plasma samples to avoid preanalytical/analytical issues with urine samples (stability of mRNA, hematuria and the selection of urine fractions, including non-centrifuged urine, urine sediments, supernatant following centrifugation and exosome preparation). We proposed that addressing the preanalytical/analytical issues of plasma samples are better to control than that of urine samples. As hemolysis serves as an analytical limitation, we aimed to isolate samples free of this factor. Hematuria is a typical marker for urological disease and is difficult to avoid as of the possible contamination of miRNAs from blood cells. A cell-free plasma could be prepared only with two centrifugation steps. miRNAs in plasma and serum samples have high stability in harsh conditions such as temperature, $\mathrm{pH}$ or long-time storage in frozen conditions (15). miRNAs are protected by RNA-binding proteins (Argonaute) and high-density lipoproteins, and are embedded inside microvesicles (16).

To increase the validity of the present study, a two-step procedure was performed: A discovery phase, including patients of an early cancer stage and a subsequent validation phase using samples from patients of different cancer stages. To the best of our knowledge, the present study is the first to obtain plasma samples from the same patient at different stages of disease for surveillance monitoring.

\section{Materials and methods}

Sample collection. Plasma samples were collected from patients with papillary BC before undergoing TURB or radical cystectomy ( $\mathrm{RC}$ ) at the Department of Urology, Charité-Universitätsmedizin Berlin between May 2014 and August 2016. In addition, we collected samples after primary tumor removal prior to the control TURB or some days after the radical cystectomy. The pathological classification of tissues was performed according to the Union of International Cancer Control 2010 (17). All patients provided written informed consent; the present study approved by the Ethics Committee of the Hospital Charité-Universitätsmedizin Berlin (EA1/134/12). The pathological classification for all BC patients is summarized in Tables I and II. The study was performed in two steps.

Firstly, a small discovery cohort of each 10 patients with (6 males, 4 females) and without ( 7 males, 3 females) BC was analyzed (Table I). The group of patients without BC included 3 healthy persons and 7 patients with other urologic diseases not related to any type of malignancy (Table I). All BC patients underwent TURB or RC. They did not received any pretreatments. In all groups patients with metastasis or any other type of malignancy were excluded from BC group. The result of the first analysis was validated in a second cohort of 36 patients (Table II) patients were distinguished according to the result of the second surgery. The first group included 13 patients (12 males, 1 female) where the tumor was completely removed during the first TURB and the second TURB after some weeks showed a non-malignant result. The second group comprised 15 patients (10 males, 5 females) with a tumor after the second TURB, and a third group included 8 patients ( 7 males, 1 female) who received RC and had postoperative plasma samples. Plasma samples were also collected prior to the second surgeries.

Sample preparation and the control of interfering hemolysis. Peripheral venous blood was collected in a $6.0 \mathrm{ml}$ K-EDTA-Vacutainer (BD Biosciences). Two centrifugation steps at room temperature prepared cell-free plasma immediately after collection. The first centrifugation step was conducted at 2,500 $\mathrm{x}$ g for $15 \mathrm{~min}$. The supernatant from this step was centrifuged at $1,500 \mathrm{x} g$ for $10 \mathrm{~min}$. Aliquots of the cell-free plasma were stored at $-80^{\circ} \mathrm{C}$. For a reliable miRNA analysis, it is important that the plasma is free from hemolysis. To exclude this interference, the absorption of plasma samples was measured at $414 \mathrm{~nm}$ using NanoDrop 1000 spectrohotometer (NanoDrop Techologies; Thermo Fisher Scientific, Inc.). Only samples with an absorption lower than 0.3 were used for further analysis (18). In addition to measuring the absorption of samples, hemolysis was assessed by determining the levels of miR-451 and miR-23a via reverse transcription-quantitative polymerase chain reaction (RT-qPCR). The first is expressed in red blood cells and the other is relatively stable in plasma and is not affected by hemolysis (19-21). Quantification cycle (Cq) differences between miR-23a and miR-451 higher than 7.0 indicate a high risk of hemolysis (19). Corresponding samples were excluded from further analysis.

$R N A$ preparation and $R T-q P C R$ of miRNAs. Exiqon Services performed all preparation and analytical steps for process in the present study, including total RNA extraction from the plasma, quality control and the RT-qPCR of miRNAs. During the discovery phase, total RNA was extracted from the plasma samples using the miRCURY ${ }^{\mathrm{TM}}$ RNA isolation kit-biofluids (Exiqon). An aliquot of $200 \mu 1$ plasma per sample was used. All following steps were performed according to the instruction manual v1.5 section B.

During the validation phase, total RNA was extracted from $200 \mu \mathrm{l}$ plasma using the miRCURY RNA isolation kit-biofluids, a high-throughput bead-based protocol v.1 (Exiqon) in an automated 96-well format. In both phases, the purified total RNA was eluted in a final volume of $50 \mu \mathrm{l}$.

In the discovery phase, $7 \mu \mathrm{l}$ RNA was reverse transcribed in a $35 \mu \mathrm{l}$ reaction volume using the miRCURY LNA ${ }^{\mathrm{TM}}$ Universal RT microRNA PCR, Polyadenylation, and cDNA synthesis kit (Exiqon). In the validation phase, the volume was reduced to $2 \mu \mathrm{l}$ RNA and $10 \mu \mathrm{l}$ reaction volume for RT. cDNA was diluted 50X and assayed in $10 \mu \mathrm{l}$ PCR reactions according to the protocol for miRCURY LNA ${ }^{\mathrm{TM}}$ Universal RT microRNA PCR. Each miR was assayed once by qPCR on the microRNA Ready-to-Use PCR, Serum/Plasma Focus panel v.4.0, including 179 microRNAs and using ExiLENT $\mathrm{SYBR}^{\circledR}$ Green master mix (Roche Diagnostics GmbH). In both phases, the qPCR reaction was performed at $95^{\circ} \mathrm{C}$ for $10 \mathrm{~min}$, followed by 45 amplification cycles at $95^{\circ} \mathrm{C}$ for $10 \mathrm{sec}$, and $60^{\circ} \mathrm{C}$ for $60 \mathrm{sec}$. The primer sequences are proprietary 
Table I. Clinicopathological characteristics of the discovery cohort of patients with and without bladder cancer.

\begin{tabular}{lcc}
\hline Features & Non-tumor & Bladder cancer \\
\hline All cases & 10 & 10 \\
Urolithiasis & 3 & \\
Cystitis cystica & 3 & \\
BPH & 1 & \\
Healthy & 3 & $48-77$ \\
Age, years & $48-85$ & 66.5 \\
Median & 65.0 & \\
Sex & & 6 \\
Male & 7 & 4 \\
Female & 3 & 10 \\
Grading & & \\
Low & & 0 \\
Tumor stage & & 0 \\
$\quad$ NMIBC (9xpTa, 1xpT1) & & \\
Distant metastasis & & \\
Lymph nodes & &
\end{tabular}

$\mathrm{BPH}$, benign prostatic hyperplasia; NMIBC, non-muscle invasive bladder tumors.

information of Exiqon Services, available from QIAGEN $\mathrm{GmbH}$. The miRNA sequences and primer sets used were listed in Table III. After selecting for reference miRNAs, the geometric mean of let-7i-5p and miR-29c-3p was used for relative quantification of RT-qPCR data in both phases. The amplification was performed using a LightCycler $^{\circledR} 480$ Real-Time PCR system (Roche $\mathrm{GmbH}$ ) in 384-well plates. The amplification curves were analyzed using the Roche LC software version 1.5.0.39, both for determination of $\mathrm{Cq}$ (by the 2nd derivative method) and for melting curve analysis (22).

Data analysis. Data were presented as median values with the interquartile range, or with the confidence interval. The miRNA levels of the Serum/Plasma Focus panel v.4.0 were assessed using the qBase ${ }^{\text {Plus }}$ (Biogazelle) software version 3.2 (20180721216) and the geNorm computer program. This software was used to identify reference miRNAs and to detect differential miRNA levels between controls and patients based on the global normalization and reference miRNA-based approaches described below. Statistical analyses and area under the curves (AUC) of receiver-operating characteristics (ROC) analyses were performed using GraphPad Prism version 5.04 for Windows (GraphPad Software, Inc.) and MedCalc Statistical Software version 18.5 (MedCalc Software bvba). Non-parametric tests, a Mann-Whitney U test for independent and a Wilcoxon test for paired samples, logistic regression analysis, $\chi^{2}$ test, Fisher's exact test, Spearman rank correlation coefficients, McNemar test and sample size calculations were applied. To evaluate the clinical validity of the miRNAs with respect to avoiding type I and type II errors, the conventional thresholds of $\alpha=0.05$ (significance level) and a power of 0.80 were selected for sample size calculations.
An internal validation of relevant results was performed using the bias-corrected and accelerated bootstrap method via SPSS 25 software (IBM Corp.) with 2,000 bootstrap replicates. Calculations concerning the within-subject and between-subject components of biological variation were conducted in consideration of the recommendations reported by Fraser (23). $\mathrm{P}<0.05$ (two-tailed) was considered to indicate a statistically significant difference.

\section{Results}

Discovery phase: Differential plasma miRNA levels between controls and BC patients. The BC cohort consisted of low grade and early stage patients (Table I). These patients were deliberately selected to establish a miRNA profile particularly for patients suffering from an early cancer stage and facilitate therefore the identification of single sensitive markers for diagnostic purposes. The control group included healthy individuals and non-tumor patients with other urological diseases (Table I) that did not exhibit differential miRNA levels. Of the total 179 miRNAs on the Exiqon panel, 122 miRNAs were detected in the 20 samples of the non-malignant and BC group. Based on the global normalization approach, the levels of nine miRNAs (Table IV) were different between the two groups.

For the reference miRNA search, the inclusion criteria included a fold change of +1.09 to -1.09 between controls and patients; $\mathrm{Cq}<31$. These criteria fulfilled 17 miRNAs of the 122 detectable miRNAs. According to these criteria, the miRNAs let-7i-5p and miR-29c-3p were identified as useful normalizers according to the geNorm approach in the software qBase ${ }^{\text {Plus }}$ (Fig. 1). The average expression stability value (M) of all candidate reference miRNA between $0.235-0.5$ denote good stability for all candidate reference miRNAs that were distinctly lower than the default limit of 1.5 in the geNorm program. The lowest M-value indicates a gene with the highest stability (Fig. 1A). Furthermore, the program calculates a normalization factor $(\mathrm{V})$, which is a criterion for determining the number of reference miRNAs. The program recommends $\mathrm{V}<0.15$ for reliable normalization. The pair of miRNAs (let-7i-5p and miR-29c-3p) achieved this cut-off value (Fig. 1B). Therefore, the geometric mean of miRNAs let-7i-5p and miR-29c-3p served as the normalization factor for calculating the differential expression levels of miRNAs between the control and BC patients.

Based on this reference miRNA approach, 13 differentially expressed miRNAs were identified between the two cohorts (Table IV). Eight of these 13 miRNAs were identical with the nine miRNAs detected using the global normalization approach. To select miRNAs for further validation, we compared the two normalization approaches and applied the following criteria: i) A fold change level higher or lower than 1.2 obtained in both normalization approaches; ii) Cq-values $<35$ in controls and patients; and iii) equal significant P-values with both normalization methods. According to these criteria, three miRNAs with elevated levels (miR-15b-5p, miR-590-5p and miR-29b-3p) and two miRNAs with reduced levels (miR-10b-5p and miR-144-5p) were selected as potential miRNA candidates for further validation in patient samples.

Validation phase: Plasma miRNA candidates as diagnostic and surveillance markers. Based on the summarized mean 
Table II. Clinicopathological characteristics of the validation cohort of patients with bladder cancer.

\begin{tabular}{lccc}
\hline Features & $\begin{array}{c}\text { TURB } \\
\text { aPatients with tumor } \\
\text { Non-malignant in follow-up }\end{array}$ & $\begin{array}{c}\text { RC } \\
\text { aPatients with tumor } \\
\text { a Malignant in follow-up }\end{array}$ & $\begin{array}{c}\text { aPatients with tumor } \\
\text { anon-malignant in follow-up }\end{array}$ \\
\hline All cases & 13 & 15 & 8 \\
Age (years) & $55-85$ & $48-87$ & $52-79$ \\
Median & 70.0 & 74.0 & 67.0 \\
Sex & 12 & 10 & 7 \\
Male & 1 & 5 & 1 \\
Female & $30-147$ & $28-365$ & $7-98$ \\
Time interval (days) & 43 & 86 & 7 \\
Median & 6 & 10 & 0 \\
Grading & 7 & 5 & 8 \\
Low & & & 0 \\
High & 13 & 13 & 8 \\
Tumor stage & 0 & 2 & 0 \\
NMIBC & 0 & 0 & 1 \\
MIBC & 0 & 0 & \\
Distant metastasis & & & \\
Lymph vessels & & & \\
\hline
\end{tabular}

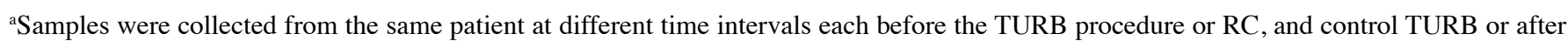
RC (follow-up). MIBC, muscle-invasive tumors; NMIBC, non-muscle invasive bladder tumors; RC, radical cystectomy; TURB transurethral resection of bladder tumor.
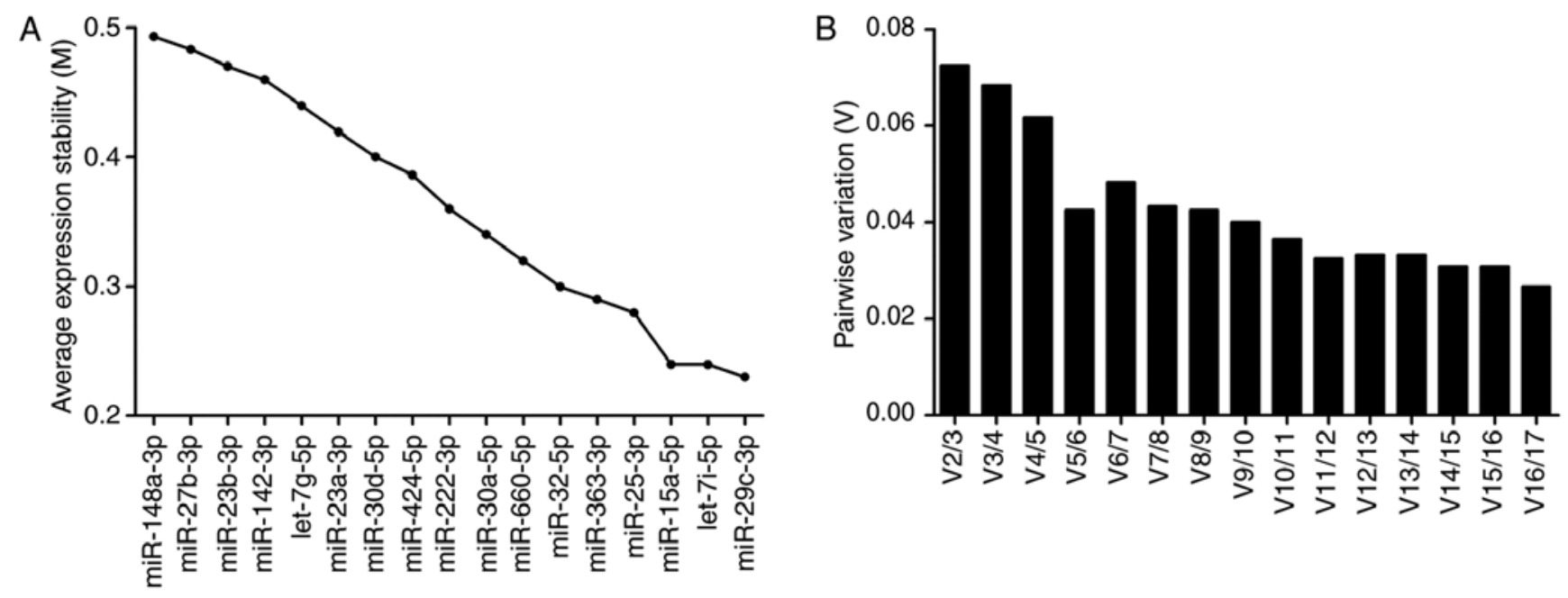

Figure 1. geNorm analysis of potential reference genes to determine the most stable genes for the normalization of miRNA expression. (A) The expression stability parameter $\mathrm{M}$ of one gene based on the average pairwise variation between all studied genes. The lowest $\mathrm{M}$-value indicates genes with the most stable expression. (B) Calculation of the optimal number of reference genes for normalization. The optimal number of reference genes in this experiment was two (hsa-let-7i-5p and has-miR-29c-3p). All results are presented according to the output files of geNorm program. miR, microRNA.

difference of the five significant candidate miRNAs between controls and $\mathrm{BC}$ patients in the discovery phase, together with the standard deviations in the two groups, the required number of cases for the validation of the discovery results were calculated with a power of 0.80 to obtain data for statistical significance based on $\alpha=0.05$. As the disease-monitoring capacity of miRNAs following tumor removal was included in the validation phase, the ratio of controls to tumor patients was altered to $1: 3$. Under these conditions, necessary sample sizes of 10 controls and 30 patients with BC were calculated. The validation cohort included $36 \mathrm{BC}$ cases, and 10 controls from the discovery phase control group included three healthy patients among non-tumor patients with other urological diseases (Table I); their values did not significantly differ from the values of healthy patients $(\mathrm{P}=0.517$ and 0.732 for miR-15b-5p and miR-590-5p, respectively; data not shown). 
Table III. miRs analyzed via reverse transcription-quantitative polymerase chain reaction.

A, miRs

Human miR miR target sequence (5'-3')

Corresponding LNA ${ }^{\mathrm{TM}}$ microRNA

PCR primer set (cat. no.)

Cat. no. ${ }^{\mathrm{a}}$

\begin{tabular}{llll}
\hline miR-29b-3p & UAGCACCAUUUGAAAUCAGUGUU & 204679 & YP00204679 \\
miR-590-5p & GAGCUUAUUCAUAAAAGUGCAG & 204222 & YP00204222 \\
miR-10b-5p & UACCCUGUAGAACCGAAUUUGUG & 205637 & YP00205637 \\
miR-15b-5p & UAGCAGCACAUCAUGGUUUACA & 204243 & YP00204243 \\
miR-144-5p & GGAUAUCAUCAUAUACUGUAAG & 204670 & YP00204670 \\
miR-29c-3p & UAGCACCAUUUGAAAUCGGUUA & 204729 & YP00204729 \\
let-7i-5p & UGAGGUAGUAGUUUGUGCUGUU & 204394 & YP00204394 \\
\hline
\end{tabular}

B, Internal RNA spike-in references

Corresponding LNA ${ }^{\mathrm{TM}}$ microRNA

\begin{tabular}{lccc} 
Reference & Sequence (5'-3') & PCR primer set (cat. no.) & Cat. no. \\
\hline UniSp2 & $-\mathrm{b}$ & 203950 & YP00203950 \\
UniSp4 & - & 203953 & YP00203953 \\
UniSP5 & - & 203955 & YP00203955 \\
cel-miR39-3p & - & 203952 & YP00203952 \\
Interplate calibrator & & Not for sale & Not for sale
\end{tabular}

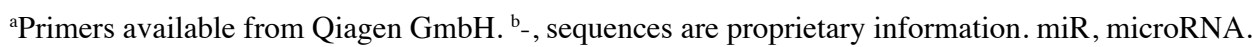

The consistent long-term quality control of the miRNA measurements performed by Exiqon justified the use of the control group for discovery as controls in the validation phase. In line with the findings from the discovery phase, the plasma levels of miR-15b-5p and miR-590-5p were significantly elevated in patients, indicating diagnostic benefit (Fig. 2). As the control group included not only healthy individuals but also patients with typical urological diseases without cancer (Table I), a selection bias could therefore be ruled out. The internal validation of data by bootstrapping supported the statistical results (Fig. 2).

The ROC curve analysis demonstrated an AUC of 0.997 [95\% confidence interval (CI), 0.918-1.000] for miR-15b-5p and an AUC of 0.774 (95\% CI, 0.626-0.884) for miR-590-5p as diagnostic biomarkers. The levels of miR-15b-5p discriminated BC patients from control subjects with a sensitivity of $97.2 \%$ and specificity of $100.0 \%$ with a Youden index of 0.9722 . For miR-590-5p, the sensitivity and specificity of miR-590-5p were 69.4 and $90.0 \%$, respectively, with a Youden index of 0.5944 . The combination of the two miRNAs based on a logistic regression model did not further improve the discrimination ability achieved by miR-15b-5p alone (Fig. 3). Increased miR-15-5p levels were evident and were also supported by bootstrapping calculations for high grade tumors and MIBCs; the levels of miR-590-5p were markedly unchanged (Fig. 4). These data correspond to Spearman rank correlation coefficients $\left(\mathrm{r}_{\mathrm{s}}\right)$ obtained between tumor stage or grade and the two miRNAs as follows: NMIBC/MIBC with miR-15b-5p $\left(\mathrm{r}_{\mathrm{s}}=0.308, \mathrm{P}=0.0677\right)$ and with miR-590-5p $\left(\mathrm{r}_{\mathrm{s}}=-0.023, \mathrm{P}=0.8930\right)$ and low/high grade with miR-15b-5p $\left(r_{s}=0.293, P=0.0823\right)$ and with miR-590 $\left(r_{s}=-0.070, P=0.6851\right)$. The results of correlation analysis for clinicopathological characteristics were summarized in Table V. The different levels of miR-29b-3p, miR-10b-5p, and miR-144-5p between tumor patients and controls found in the discovery phase could not be confirmed.

To assess the validity of the five selected miRNAs as surveillance biomarkers, the $36 \mathrm{BC}$ patients were classified into three groups according to the tumor presence in the control TURB specimens and the collection after RC (Table II). Fig. 5 presented the expression levels of the two potentially diagnostic miRNAs in the three groups. In 13 patients, the bladder tumor was completely removed at the first TURB, while non-malignant results were reported for the second control TURB (Fig. 5A). In the second group with 15 patients, malignant lesions were detected in the control TURB after a median follow-up of 86 days (Fig. 5B). Plasma samples were also collected from 8 patients a few days after the radical cystectomy (Fig. 5C). None of the five selected miRNAs for validation showed any differences between tumor samples before the first TURB, and follow-up samples before the control TURB or after RC from the same patient. Paired Wilcoxon analyses revealed that the levels of miRNAs in the plasma were not statistically different in patients with non-malignant or malignant histological results after TURB compared with the data before the treatment (Fig. 5A and B). After BC removal, the expected reduction of miR-15b-5p and miR-590-5p miRNA levels occurred only in 7 and 8 of 13 patients, respectively (Fig. 5A). Similarly, notably 
Table IV. Differences in the expression levels of miRs in plasma samples of patients with bladder cancer compared with the control group of discovery cohort.

\begin{tabular}{|c|c|c|c|c|c|}
\hline \multirow[b]{3}{*}{$\mathrm{miR}$} & \multicolumn{4}{|c|}{ Normalization } & \multirow[b]{3}{*}{$\mathrm{Cq}$ (range) } \\
\hline & \multicolumn{2}{|c|}{ Two reference miRNAs } & \multicolumn{2}{|c|}{ Global mean } & \\
\hline & P-value & Fold change & P-value & Fold change & \\
\hline has-miR-19a-3p & 0.01 & 1.25 & 0.08 & 1.24 & $25.1-28.2$ \\
\hline hsa-miR-29b-3p & 0.01 & 1.33 & 0.02 & 1.32 & $29.6-32.8$ \\
\hline hsa-miR-590-5p & 0.01 & 1.34 & 0.03 & 1.33 & $30.5-33.3$ \\
\hline has-miR-2110 & 0.02 & -2.26 & 0.02 & -2.28 & 34.6-ND \\
\hline has-miR-144-3p & 0.02 & 1.30 & 0.11 & 1.29 & $24.8-27.1$ \\
\hline hsa-miR-144-5p & 0.02 & -1.85 & 0.02 & -1.86 & $29.5-33.8$ \\
\hline has-let-7b-3p & 0.03 & -2.01 & 0.01 & -2.02 & $33.2-37.2$ \\
\hline has-miR-19b-3p & 0.04 & 1.24 & 0.13 & 1.24 & $23.8-27.4$ \\
\hline has-miR-425-5p & 0.04 & 1.20 & 0.15 & 1.19 & $28.2-31.5$ \\
\hline hsa-miR-10b-5p & 0.04 & -1.46 & 0.04 & -1.47 & $30.9-33.1$ \\
\hline has-miR-324-5p & 0.04 & 1.41 & 0.03 & 1,41 & 31.7-ND \\
\hline has-miR-93-5p & 0.05 & 1.33 & 0.08 & 1.32 & $26.3-30.4$ \\
\hline hsa-miR-15b-5p & 0.05 & 1.57 & 0.02 & 1.57 & $29.6-33.1$ \\
\hline has-miR-29a-3p & 0.09 & -1.32 & 0.03 & -1.32 & $29.7-32.1$ \\
\hline
\end{tabular}

$\mathrm{P}<0.05$, marked in bold font, including miRs with equal $\mathrm{P}$-values following both normalization methods, and Cq $<35$. Hsa, homo sapiens; miRs, microRNA; ND, not detectable.

decreased or unchanged miRNA levels were observed when the malignant lesions were detected in the follow-up control TURB. Considering that the interval between the first and the second sampling could be too short, which may account for these unexpected values, a time-dependent regression calculation of the miRNA levels in the two TURB cohorts was performed. A time-dependent effect was not observed (Fig. S1). Furthermore, both TURB cohorts did not differ in their tumor aggressiveness (tumor grade and tumor stage: Fisher's exact test with $\mathrm{P}=0.445$ and 1.000 , respectively). In addition, in the cohort with the remaining tumor, tumor grade and tumor stage did not differ in the paired samples in the first and second TURB specimen (McNemar analysis, $\mathrm{P}=1.000$ ). We also conducted multivariate logistic regression analysis for clinical parameters, tumor stage and grade, and the plasma levels of both miRNAs between the first and second TURB, but did not improve the prediction of complete tumor removal. Only the tumor stage remained as independent variable (data not shown). A change in the miRNA level was not observed a few days after radical cystectomy (Fig. 5C). In all cases, these statistically unchanged miRNA patterns could not be attributed to obvious clinical manifestations.

To support these statistical data, we investigated the effects of within-subject or intra-individual biological variation of miR-15b-5p and miR-590-5p in these two time-dependent measurements of our study. For that purpose, the database from Ammerlaan and Betsou (24), which summarized the within-subject variation of numerous miRNAs in blood plasma and serum, was used. For example, miR-15b-5p was characterized by a mean coefficient of variation of $\sim 65 \%$. Considering an analytical variation of only $10 \%$, the reference change value (also known as 'critical values' between serial data) of $\sim 180 \%$ would result; $95 \%$ of the dispersions of miR-15b-5p (and also of miR-590-5p) of the two values of the patients considerably overlapped due to this inherent intra-individual biological variation. Therefore, the results were not significantly different. Monitoring of the follow-up of disease would be hardly possible using these miRNAs due their high biological variation, resulting in a large fluctuation of the miRNA levels.

\section{Discussion}

miRNAs have been proposed as optional novel biomarkers in a variety of diseases $(6,25)$. miRNAs may be applied as sensitive and specific tools for diagnostic, prognostic and predictive purposes, particularly in different types of cancer $(26,27)$. The urgent need for reliable non-invasive markers for patients with $\mathrm{BC}$ has been emphasized as an important demand in the European Association of Urology guidelines (2). However, miRNAs in the blood plasma from BC patients have been examined in only a few studies, exclusively as diagnostic biomarkers $(8-11,13)$. Thus, we performed this study with aim to provide insight into miRNAs as diagnostic tools and investigate the monitoring capacity of miRNAs with strict control of potential interfering effect of hemolysis in plasma samples. miR-15b-5p and miR-590-5p were finally confirmed in the validation phase, based on five potential miRNAs candidates identified in the primary discovery phase, as significant discriminative markers between patients with $\mathrm{BC}$ and controls. However, none of these miRNAs were determined to be suitable for monitoring the disease. 

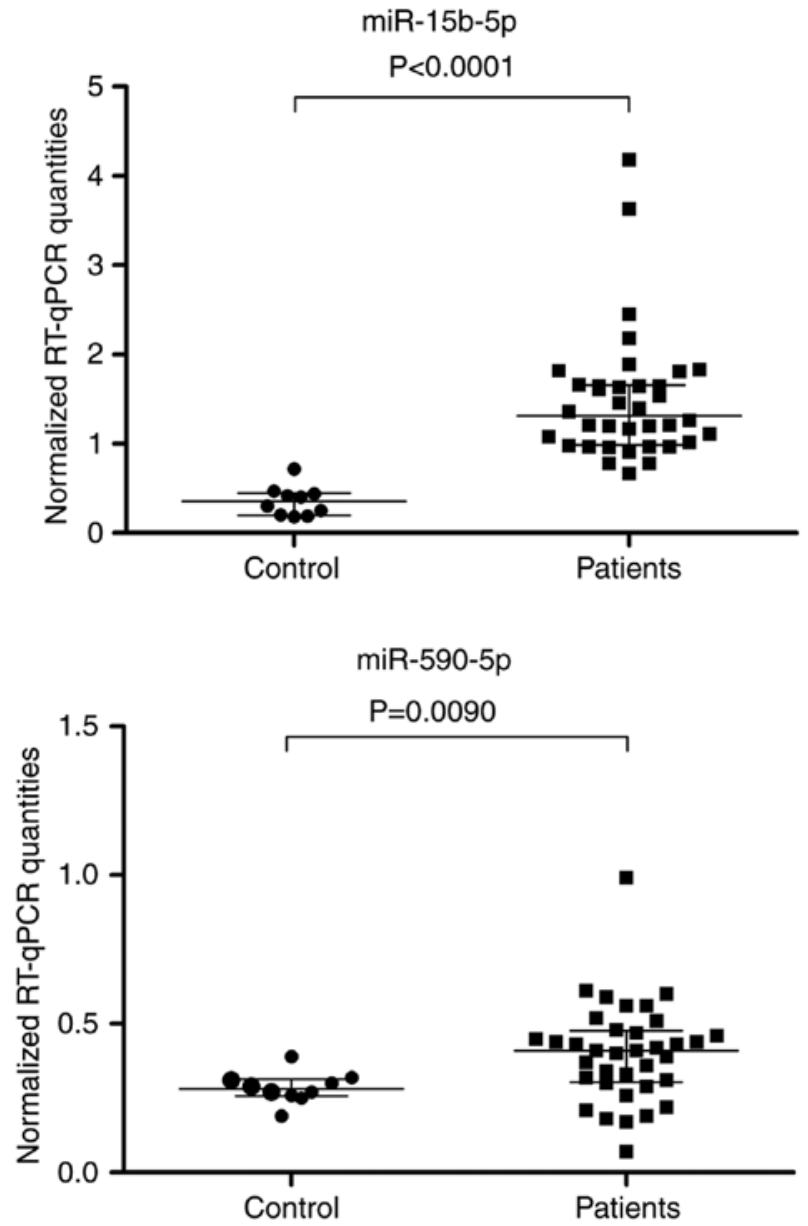

Figure 2. miR-15b-5p and miR-590-5p levels in plasma samples of controls and patient with bladder cancer. miRNA levels were measured with RT-qPCR, and normalized with miR-29c-3p and let-7i-5p. Patient samples that were histologically confirmed for tumor were collected before transurethral resection or radical cystectomy. All tumor stages are included. The graphs show the median with interquartile range. Significances were calculated between control and patients using Mann-Whitney U-test in case of miR-15b-5p bootstrap calculations (2,000 re-samples) confirmed the statistical results of the Mann-Whitney U-test showing $(\mathrm{P}<0.0001$ for miR-15-5p and $\mathrm{P}=0.0015$ for miR-590-5p, respectively). miR, microRNA; RT-qPCR, reverse transcription-quantitative polymerase chain reaction.

To the best of our knowledge, neither plasma miR-15b-5p or miR-590-5p have been reported in previous studies with regards patients with $\mathrm{BC}$ (8-13). Previous studies revealed a total of 22 miRNAs (Table SI) as potential significant diagnostic markers but only three miRNAs (miR-92a-3p, miR-99 and miR-100) were reported by at least two working groups. Four miRNAs (miR-25-3p, miR-33b-5p, miR-92a-3p and miR-194) may be influenced by hemolysis or could detectable in blood cells $(20,28)$. Due to a lack of consistent results, none of these miRNA panels has been applied in practice. The present study reported the degree of hemolysis with two methods (absorption measurement and the detection of specific miRNAs) and rigorously disclosed hemolyzed plasma samples.

miR-15-5p and miR-590-5p were determined to be upregulated in $\mathrm{BC}$ in a previous own study (29) and confirmed by others (30-32). Armstrong et al (33) reported on the top 25 miRNAs with highest concentrations in BC tissue, white-blood cells, urine and plasma only in patients with $\mathrm{BC}$ but not in controls. In tissue and urine samples, the authors

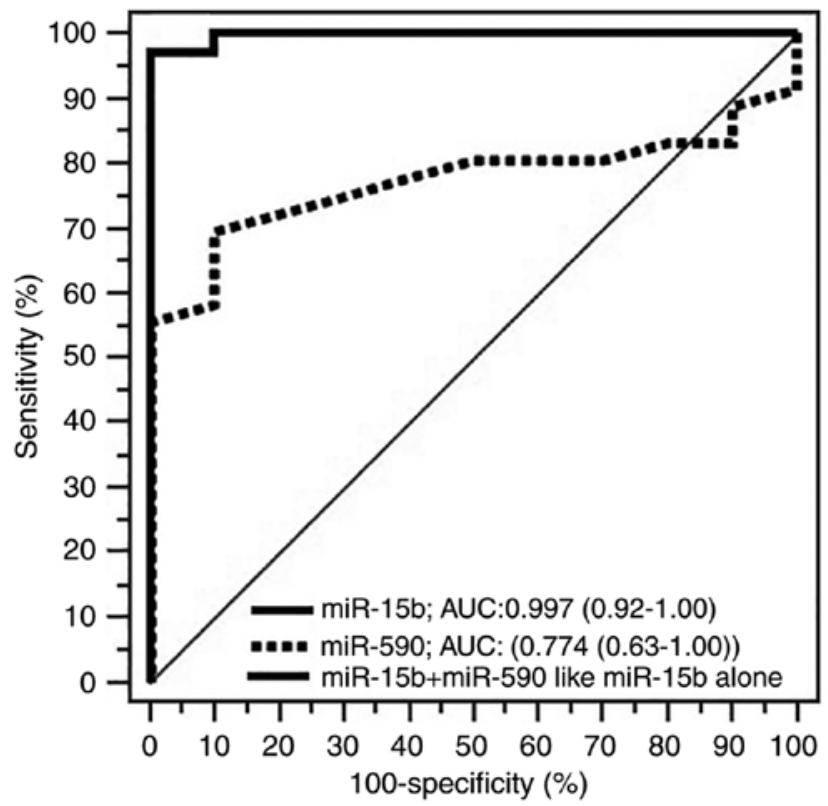

Figure 3. Receiver-operating characteristic curve analysis using plasma miR-15b-5p, miR-590-5p, and the combination of both miRNAs based on a logistic regression model to differentiate bladder cancer patients from normal subjects. The combination of both miRNAs had the same AUC value as miR-15b-5p alone. AUC, area under the curve; miR, microRNA.

detected miR-15b-5p in the aforementioned top 25 miRNA whereas miR-15b-5p in plasma samples was not among the top 25 miRNAs. However, it should be considered that Armstrong et al (33) did not compare the data between controls and patients. Thus, the plasma concentration of miR-15b-5p between $\mathrm{BC}$ patients remained unknown in the report of Armstrong et al (33), whereas our data reflect the different levels between healthy individuals and BC patients. In addition, in the plasma samples of our study for the discovery phase, 122 miRNAs were detected, while miR-15b-5p was the 53rd miRNA ranked in tumor patients and 64th in controls according to the measured levels. However, as shown in Table IV, this miRNA had the highest increase in expression in cancer patients compared with controls. Thus, this result corresponds with the findings of Armstrong et al (33) in which miR-15b-5p was not observed in the top 25 miRNAs with the highest abundance in the plasma. This phenomenon of non-concordance between tissue and circulating miRNA levels was particularly evident in the study of Xie et al (34). These authors reported diagnostic markers for $\mathrm{BC}$ based on a meta-analysis of studies of tissue data of BC. The meta-analysis included 26 publications; 21 were related to tissue, four to urine and only one to serum. Therefore, the suggested potentially diagnostic miRNAs may be used for the differentiation of tissue material. The were notable discrepancies between the six common differentially expressed miRNAs in $\mathrm{BC}$ tissue of the meta-analysis by Xie et al (34); none of them were found in our analysis of plasma samples. This phenomenon termed distortion between cellular and circulating markers (35) reflects the lack of concordance in changes of circulating miRNAs with tissue expression levels described in other studies $(36,37)$. The possible explanation for this are non-uniform releasing processes of miRNAs by cells (38). The levels of miR-15b-5p were increased by 1.2 - and 2.4 -fold 
A

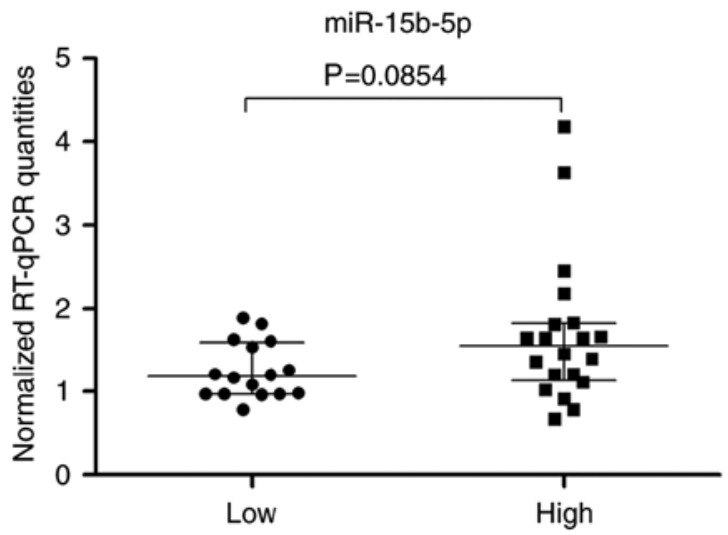

B

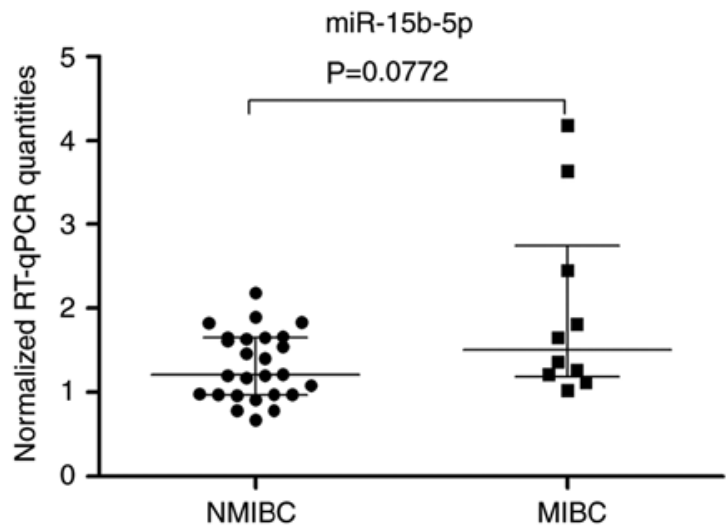

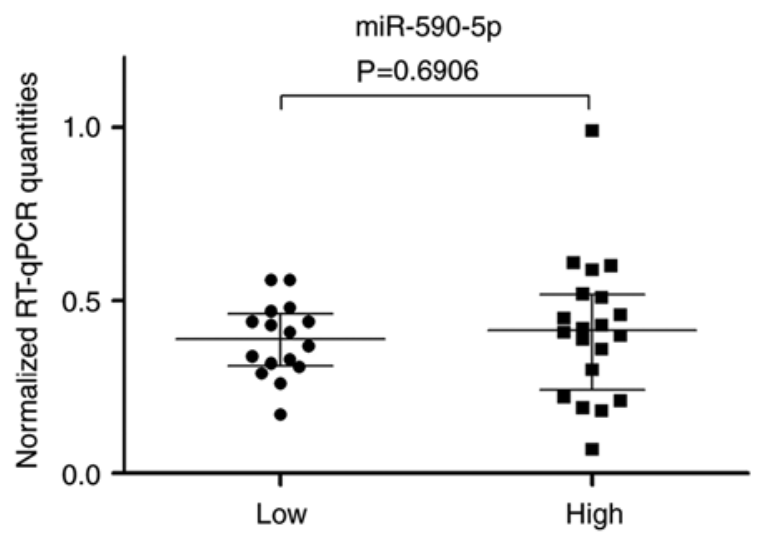

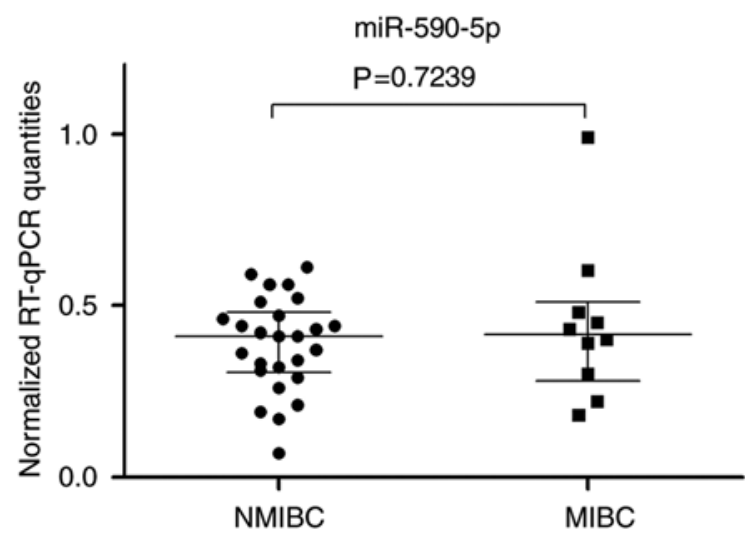

Figure 4. miR-15b-5p and miR-590-5p levels in plasma samples of bladder cancer patients depending on tumor stage and grade. miRNA levels were measured via reverse transcription-quantitative polymerase chain reaction, and normalized with miR-29c-3p and let-7i-5p. Samples was collected before transurethral resection or radical cystectomy. The patients were classified according to their (A) tumor grade (low and high grade) and (B) their tumor stage (NMIBC and $\mathrm{MIBC})$. Significances were calculated between the two groups using a Mann-Whitney U-test. Bootstrap calculations (2,000 re-samples) resulted in $\mathrm{P}=0.069$ for the low vs. high grade group and $\mathrm{P}=0.120$ for NMIBC vs. MIBC, respectively. MIBC, muscle-invasive tumors; miR, microRNA; NMIBC, non-muscle invasive bladder tumors.

Table V. Spearman correlation coefficient between miR and clinicopathological characteristics.

Validation cohort $\mathrm{n}=36$

Features miR-15b-5p miR-590-5p

\section{Sex}

rs

P-value

$-0.1740$

0.3108

$-0.0800$

0.6410

Age

rs

P-value

$-0.2360$

0.1990

0.1655

0.2442

Grade (low/high)

$\begin{array}{lll}\text { rs } & 0.2930 & 0.0700 \\ \text { P-value } & 0.0823 & 0.6851\end{array}$

\section{Stage (NMIBC/MIBC)}

\begin{tabular}{lll} 
rs & 0.3080 & 0.0230 \\
P-value & 0.0677 & 0.8930 \\
\hline
\end{tabular}

MIBC, muscle-invasive tumors; miR, microRNA; NMIBC, non-muscle invasive bladder tumors; rs, Spearman rank correlation coefficient. in urothelial carcinoma than in adjacent normal bladder tissue $(29,30)$. Furthermore, based on bioinformatics analysis of The Cancer Genome Atlas, Wang et al (32) reported a relationship between miR-15b-5p and age-associated genes in $\mathrm{BC}$. This is interesting as the incidence of $\mathrm{BC}$ increases after the 40th year of life and reaches a maximum in the sixth and seventh decade (39). The examined patient collective were aged in their $60 \mathrm{~s}$ and $70 \mathrm{~s}$ in the analysis conducted by Wang et al (32). Moreover, the long-life expectancy in western countries has been linked to an increase in BC cases as the risk of disease increases with age (1). The expression of the other relevant miRNA miR-590-5pwas determined to be 2.98- and 3.14-fold higher in malignant than in non-malignant bladder tissue $(29,31)$. Thus, these tissue expression data support that as of the altered levels of these miRNAs due to the extent of their release from carcinoma tissue, these miRNAs may serve as potential circulating biomarkers (38). Under the stringent conditions of the present study, the diagnostic aspect of the increased levels of miR-15b-5p and miR-590-5p in the plasma of $\mathrm{BC}$ patients could be confirmed by further validation and may be an indication for the presence of $\mathrm{BC}$. In addition, our study reported that the tendency of increased levels of miR-15b-5p depending on the aggressiveness of the cancer supported its potential as a cancer biomarker. Furthermore, miR-15b was identified as a diagnostic marker in urine (40). 
A

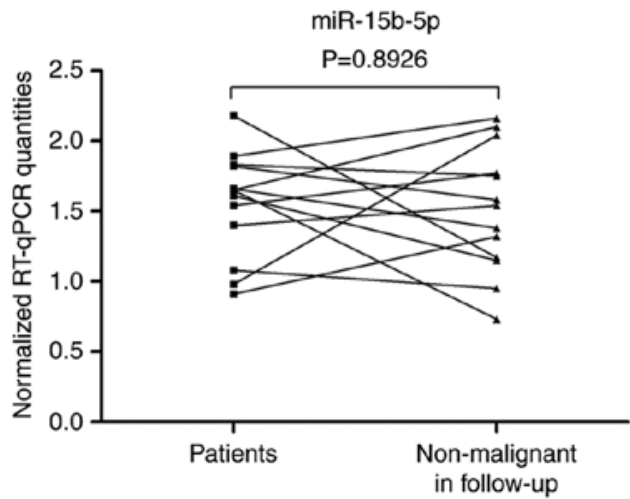

B

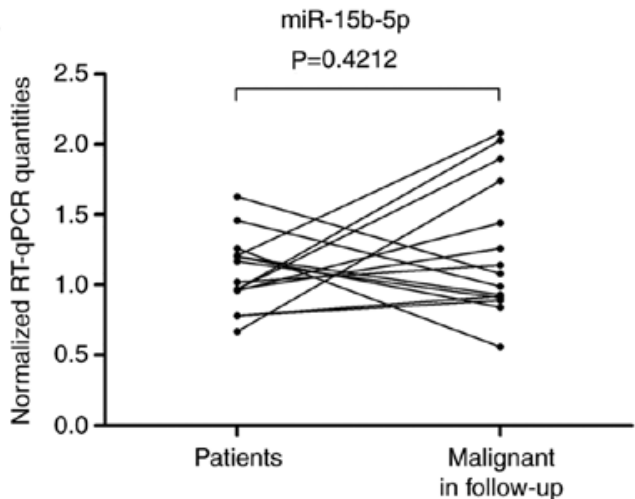

C

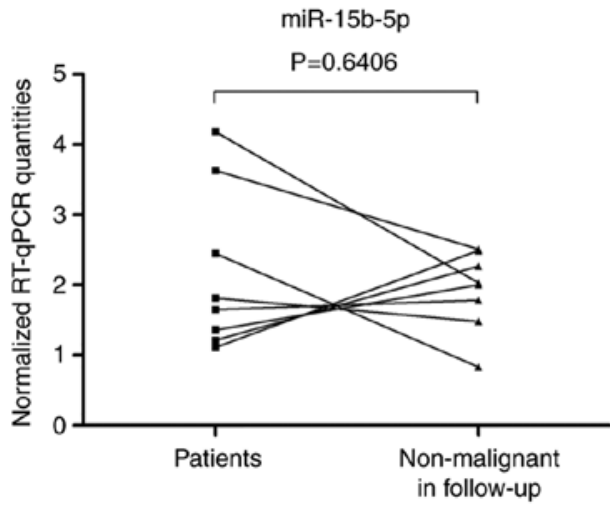

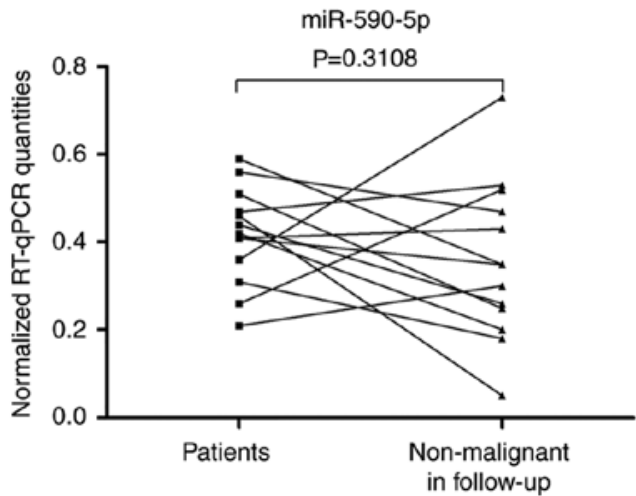
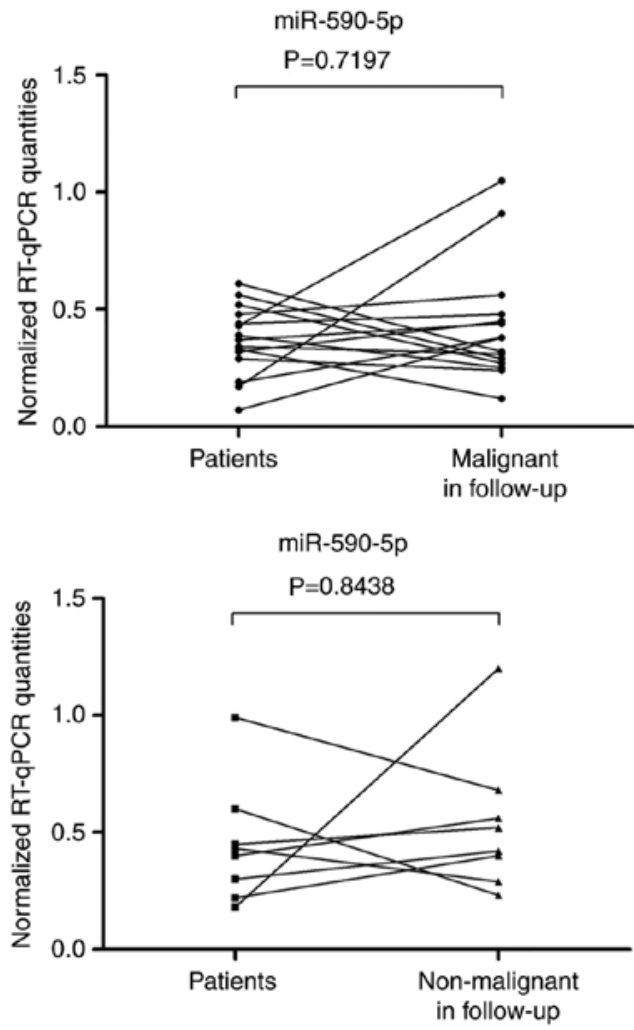

Figure 5. miR-15b-5p and miR-590-5p levels in plasma samples of bladder cancer patients in the follow-up after the first TURB. miR levels were measured with reverse transcription-quantitative polymerase chain reaction, and normalized with miR-29c-3p and let-7i-5p. Samples of patients were collected before TURB who were histologically confirmed for tumor and during follow-up before control TURB. Analysis revealed (A) the complete removal of the tumor (median follow-up: 43 days) or (B) the continuing tumor presence (median follow-up: 86 days), and (C) in samples of patients before radical cystectomy and during follow-up (median) 7 days after surgery. Statistical analysis was conducted between cancer patients and the follow-up of the same patient using a Wilcoxon test. miR, microRNA; TURB, transurethral resection.

Jiang et al (41) also reported on the use of miR-15b-5p with a decreased level in serum of patients, although it is recommended that carefully prepared plasma samples from selected specimens should be used for the quantification of circulating miRNAs (42). Therefore, we proposed that this discrepancy could be a result of a hemolysis-impaired normalization approach as Jiang et al (41) used miR-16-5p, in addition to miR-193-5p, for normalization. These miRNAs was typically found to be increased in samples that could be characterized as hemolytic by hemoglobin sensitivity measurements or the ratio $\mathrm{miR}-451 / \mathrm{miR}-23 \mathrm{a}$, whereas the effects of hemolysis are difficult to observe $(20,21)$. Furthermore, the use of different RNA isolation kits and different PCR systems could not be excluded as other analytical reasons for discrepancies in the levels of miR-15-5p in our study and that by Jiang et al (41). On the other hand, comparable increases in plasma miR-15b-5p of NMIBC and MIBC patients, and the use of a similar patient cohort to Jiang et al (41) support the conclusion that pre-analytical/analytical reasons are may be more likely to explain for these different expression levels between the studies, than biological reasons. Other researchers have detected miR-15b-5p in plasma samples in relation to other tumor types, such as colorectal carcinomas (43), hepatocellular carcinoma (44) and melanoma (45).

Few studies have reported the potential functions of miR-15b and miR-590-5p, especially in bladder cancer. Wang et al (46) detected an increased level of miR-15b-5p relative to the decreased expression of long noncoding RNA MAGI2-AS3 and the tumor suppressor CCDC19 in BC tissues and cell lines. Transfection experiments with cell cultures 
indicated miR-15b-5p as a potential an oncogene in BC (46). Similarly, overexpression of miR-15b-5p in gastric cancer was associated with the expression of the tumor suppressor progestin and adipoQ receptor family member 3 (47). Furthermore, the process of epithelial-mesenchymal transition was promoted (47).

To the best of our knowledge regarding miR-590-5p in plasma, only one report exists for patients undergoing chemoradiotherapy for the treatment of head and neck cancer (48). miR-590-5p was described as part of a six-miRNA panel that reflects the response to chemoradiotherapy (48). One target of the miR-590-5p is Yes associated protein (YAP) (49), which induces cell growth and invasion in $\mathrm{BC}(50)$. Overexpression of YAP is partly responsible for poor prognosis in $\mathrm{BC}(50,51)$. Furthermore, for NMIBC, epigenetic regulation of the FGF gene family was described (52), which is involved in a variety of biological processes, including tumor growth (53). Zhang et al (53) revealed that FGF18 is negatively regulated by miR-590-5p, which leads to a promotion of gastric tumorigenesis.

In terms of disease follow-up, we assumed that the dysregulated miRNAs in plasma samples from BC patients may return to the levels recorded for the non-tumor samples after some time. In the present study, the levels of the five selected miRNAs in the plasma samples, particularly miR-15b-5p and miR-590-5p, did not independently return to that for the control after the second TURB. This suggested these two miRNAs as potential diagnostic biomarkers. The median time of 50 days corresponds to the usual time interval between the first and second TURB (2); 7 days after RC, the miRNA levels had not yet returned to normal. Time-dependent regression line calculations of the miRNA levels measured before the control TURB and percentage related to the levels before the first TURB, did not show a time-dependent effect. The half-lives of different cellular miRNAs differ between 1 and $120 \mathrm{~h}$ (54-56). Based on these data, it could be assumed that circulating miRNAs released by cancer cells may rapidly degrade or at least notably decrease in their levels after cancer removal. In BC patients, a tumor reversal based on miRNA changes was observed only for urinary cell-free miRNAs (57-59). A postoperative decrease of circulating miRNAs was also reported in hepatocellular, gastric, colorectal, lung and breast cancer within 7-10 days after surgery $(16,44,60-62)$. However, compared with the preoperative levels of tumor-associated miRNAs in lung and prostate carcinoma, both unchanged and decreased miRNA levels, depending on specific miRNAs and the cancer type, were observed after surgery $(61,63-66)$. This phenomenon of statistically comparable pre-and postoperative miRNA levels corresponds with our results.

Additionally, a selection bias is very unlikely for this contradictory observation in the clinical use as diagnostic and monitoring markers in our case-control study. However, the relatively high biological variation in comparison to the analytical variation of the two miRNAs should be considered as one essential reason for this phenomenon. The result is an overlap of the $95 \%$ dispersion of the repeated values and the lack of statistical significance. A similar fluctuating miRNA pattern was measured in blood samples collected before, and 7-17 days and six subsequent 3 month intervals after lung cancer resection (64). This effect may impair the clinical validity of biomarkers particularly for monitoring purposes (23). At present, only scarce data $(24,67)$ are available regarding the within-subject variability of circulating miRNA levels to support this view. However, we believe that this aspect in the application of circulating miRNAs as monitoring markers requires further investigation. This issue requires greater attention in the future, taking into account the recent recommendations for evaluating studies on biological variation (68).

Certain limitations of the present study should be addressed, including the low number of included patients, their short follow-up times, and the different composition of the discovery and validation cohorts with regard to tumor stage and grade. To avoid possible type I and type II errors with the low number of patients, we performed an additional internal validation using a bootstrapping approach with confirming results. The limited number of patients was evident for the lack of statistically significant associations between the increased levels of miR-15b-5p depending on the cancer aggressiveness. As more patients could not be recruited, we believe that the data of the post hoc power analysis are appropriate to support this influencing effect. To further investigate this, a post hoc power analysis was conducted based on these population-related effect sizes of median differences, the study cohort proportions, and a power of 0.80 to obtain data for statistically significant results on a level of $\alpha=0.05$. For the low/high tumors, NMIBCs and MIBCs, the participants of the present study would have to increase from 36 to 72 and 94 , respectively. It could be criticized that the time interval between the first and second TURB was too short to achieve stable circulating miRNA levels in plasma. This interval corresponds to the regular follow-up examinations within the risk management of these patients. In addition, the time-dependent regression line calculations could not confirm this concern of a time-dependent effect. Furthermore, there are differences in patient cohorts in the discovery and validation phase. The discovery phase included only low grade tumors and NMIBCs while the validation phase included high grade tumors and MIBCs. However, as explained in the objective of our study, this design was deliberately selected in the discovery phase in order to identify potential diagnostic markers already in early cancer stages.

In conclusion, despite these limitations but based on the selected stringent pre-analytical and analytical conditions, and the evident statistical significances of the two circulating miRNAs, miR-15b-p and miR-590-5p could be verified as discriminative biomarkers between cancer cases and controls to provide an initial indication of a possible tumorigenic disease. Further diagnostic methods must confirm our first hypothesis. However, for the surveillance of $\mathrm{BC}$ patients, neither of the investigated miRNAs seem to be informative. An expected postoperative decline of the levels of miR-15b-5p and miR-590-5p as the miRNAs of interest could not be observed in some patients after tumor removal. This contradictory behavior of miRNA patterns could not be attributed to any notable or latent clinical manifestations. On the other hand, calculations of the 'critical differences' (23) of the miRNA levels in the follow-up reported in our study may explain this phenomenon caused by a high biological variation of the examined circulating miRNAs. Similar patterns have been described in the literature and confirm our observations $(61,63-66)$. Our findings support the view that diagnostic markers are by no means 
always suitable as monitoring markers (69); however, current knowledge of the effects of biological variation of circulating miRNAs is markedly limited. Particular attention and further research to this critical point will be necessary to evaluate the true validity of circulating miRNAs in clinical practice. Further prospective investigations of circulating miRNAs are warranted to consider the aforementioned issues for a suitable study design.

\section{Acknowledgements}

Not applicable.

\section{Funding}

The project was supported by Stiftung Urologische Forschung.

\section{Availability of data and materials}

The datasets used and/or analyzed during the current study are available from the corresponding author on reasonable request.

\section{Author's contribution}

AT, LB and KJ were responsible for the conceptualization, investigation, methodology, validation, visualization and writing of the manuscript. AT and KJ contributed to the data curation, formal analysis of the data, and project administration. LB and KJ were involved in the funding acquisition, and carried out the writing and editing of the manuscript. AT was responsible for the data acquisition, including reagents, materials, patients and laboratory samples; and supervision of the research was conducted by KJ. All authors read and approved the final manuscript.

\section{Ethics approval and consent to participate}

All patients provided their informed consent to this study, which was approved by the Hospital Charité-Universitätsmedizin Berlin (EA1/134/12; Berlin, Germany).

\section{Patient consent for publication}

Not applicable.

\section{Competing interests}

The authors declare that they have no competing interests.

\section{References}

1. Siegel RL, Miller KD and Jemal A: Cancer statistics, 2017. CA Cancer J Clin 67: 7-30, 2017.

2. Babjuk M,BöhleA,Burger M, Capoun O,Cohen D,Comperat EM, Hernandez V, Kaasinen E, Palou J, Roupret M, et al: EAU Guidelines on Non-Muscle-invasive urothelial carcinoma of the bladder: Update 2016. Eur Urol 71: 447-461, 2017.

3. Pandith AA, Shah ZA and Siddiqi MA: Oncogenic role of fibroblast growth factor receptor 3 in tumorigenesis of urinary bladder cancer. Urol Oncol 31: 398-406, 2013.

4. Wu XR: Biology of urothelial tumorigenesis: Insights from genetically engineered mice. Cancer Metastasis Rev 28: 281-290, 2009.
5. Kim WJ and Bae SC: Molecular biomarkers in urothelial bladder cancer. Cancer Sci 99: 646-652, 2008.

6. Etheridge A, Lee I, Hood L, Galas D and Wang K: Extracellular microRNA: A new source of biomarkers. Mutat Res 717: 85-90, 2011.

7. Zen K and Zhang CY: Circulating microRNAs: A novel class of biomarkers to diagnose and monitor human cancers. Med Res Rev 32: 326-348, 2012.

8. Adam L, Wszolek MF, Liu CG, Jing W, Diao L, Zien A, Zhang JD, Jackson D and Dinney CP: Plasma microRNA profiles for bladder cancer detection. Urol Oncol 31: 1701-1708, 2013.

9. Feng Y, Liu J, Kang Y, He Y, Liang B, Yang P and Yu Z: miR-19a acts as an oncogenic microRNA and is up-regulated in bladder cancer. J Exp Clin Cancer Res 33: 67, 2014.

10. Feng Y, Kang Y, He Y, Liu J, Liang B, Yang $\mathrm{P}$ and $\mathrm{Yu} Z$ : microRNA-99a acts as a tumor suppressor and is down-regulated in bladder cancer. BMC Urol 14: 50, 2014.

11. Du M, Shi D, Yuan L, Li P, Chu H, Qin C, Yin C, Zhang Z and Wang M: Circulating miR-497 and miR-663b in plasma are potential novel biomarkers for bladder cancer. Sci Rep 5: 10437, 2015.

12. Fang Z, Dai W, Wang X, Chen W, Shen C, Ye G and Li L: Circulating miR-205: A promising biomarker for the detection and prognosis evaluation of bladder cancer. Tumour Biol 37: 8075-8082, 2016

13. Motawi TK, Rizk SM, Ibrahim TM and Ibrahim IA: Circulating microRNAs, miR-92a, miR-100 and miR-143, as non-invasive biomarkers for bladder cancer diagnosis. Cell Biochem Funct 34: 142-148, 2016.

14. Tölle A, Blobel CC and Jung K: Circulating miRNAs in blood and urine as diagnostic and prognostic biomarkers for bladder cancer: An update in 2017. Biomark Med 12: 667-676, 2018.

15. Chen X, Ba Y, Ma L, Cai X, Yin Y, Wang K, Guo J, Zhang Y, Chen J, Guo X, et al: Characterization of microRNAs in serum: A novel class of biomarkers for diagnosis of cancer and other diseases. Cell Res 18: 997-1006, 2008.

16. Huang Z, Huang D, Ni S, Peng Z, Sheng W and Du X: Plasma microRNAs are promising novel biomarkers for early detection of colorectal cancer. Int J Cancer 127: 118-126, 2010.

17. Wittekind $\mathrm{C}$, Asamura $\mathrm{H}$ and Sobin LH: TNM Atlas. Hoboken, New Jersey, US, Wiley-Backwell Publishing, 2014.

18. Shah JS, Soon PS and Marsh DJ: Comparison of methodologies to detect low levels of hemolysis in serum for accurate assessment of serum microRNAs. PLoS One 11: e0153200, 2016.

19. Blondal T, Jensby NS, Baker A, Andreasen D, Mouritzen P, Wrang Teilum $M$ and Dahlsveen IK: Assessing sample and miRNA profile quality in serum and plasma or other biofluids. Methods 59 (Suppl): S1-S6, 2013.

20. Pritchard CC, Kroh E, Wood B, Arroyo JD, Dougherty KJ, Miyaji MM, Tait JF and Tewari M: Blood cell origin of circulating MicroRNAs: A cautionary note for cancer biomarker studies. Cancer Prev Res (Phila) 5: 492-497, 2012.

21. Kirschner MB, Edelman JJ, Kao SC, Vallely MP, van Zandwijk N and Reid G: The impact of hemolysis on cell-free microRNA biomarkers. Front Genet 4: 94, 2013

22. Livak KJ and Schmittgen TD: Analysis of relative gene expression data using real-time quantitative PCR and the 2(-Delta Delta C(T)) method. Methods 25: 402-408, 2001.

23. Fraser CG: Biological variation-from principles to practice. Washington, DC, U.S., AACC Press, 2001.

24. Ammerlaan $\mathrm{W}$ and Betsou F: Intraindividual temporal miRNA variability in serum, plasma, and white blood cell subpopulations. Biopreserv Biobank 14: 390-397, 2016.

25. Ciesla M, Skrzypek K, Kozakowska M, Loboda A, Jozkowicz A and Dulak J: MicroRNAs as biomarkers of disease onset. Anal Bioanal Chem 401: 2051-2061, 2011.

26. Wang W, Peng B, Wang D, Ma X, Jiang D, Zhao J and Yu L: Human tumor microRNA signatures derived from large-scale oligonucleotide microarray datasets. Int J Cancer 129: 1624-1634, 2011.

27. Huang X, Liang M, Dittmar R and Wang L: Extracellular microRNAs in urologic malignancies: Chances and challenges. Int J Mol Sci 14: 14785-14799, 2013.

28. MacLellan SA, MacAulay C, Lam S and Garnis C: Pre-profiling factors influencing serum microRNA levels. BMC Clin Pathol 14: 27, 2014.

29. Ratert N, Meyer HA, Jung M, Lioudmer P, Mollenkopf HJ, Wagner I, Miller K, Kilic E, Erbersdobler A, Weikert S, et al: miRNA profiling identifies candidate mirnas for bladder cancer diagnosis and clinical outcome. J Mol Diagn 15: 695-705, 2013. 
30. Song T, Xia W, Shao N, Zhang X, Wang C, Wu Y, Dong J, Cai W and $\mathrm{Li} \mathrm{H}$ : Differential miRNA expression profiles in bladder urothelial carcinomas. Asian Pac J Cancer Prev 11: 905-911, 2010.

31. Zhao F, Ge YZ, Zhou LH, Xu LW, Xu Z, Ping WW, Wang M, Zhou CC, Wu R and Jia RP: Identification of hub miRNA biomarkers for bladder cancer by weighted gene coexpression network analysis. Onco Targets Ther 10: 5551-5559, 2017.

32. Wang $C$, Chen L, Yang $Y$, Zhang $M$ and Wong $G$ : Identification of bladder cancer prognostic biomarkers using an ageing gene-related competitive endogenous RNA network. Oncotarget 8: 111742-111753, 2017.

33. Armstrong DA, Green BB, Seigne JD, Schned AR and Marsit CJ: MicroRNA molecular profiling from matched tumor and bio-fluids in bladder cancer. Mol Cancer 14: 194, 2015.

34. Xie Y, Ma X, Chen L, Li H, Gu L, Gao Y, Zhang Y, Li X, Fan Y, Chen J, et al: MicroRNAs with prognostic significance in bladder cancer: A systematic review and meta-analysis. Sci Rep 7: 5619, 2017.

35. Friedel $R$, Diederichs $F$ and Lindena $J$ : Advances in clinical enzymology. Basel, S. Karger AG, Switzerland, 1979, 70-105.

36. Hauser S, Wulfken LM, Holdenrieder S, Moritz R, Ohlmann CH, Jung V, Becker F, Herrmann E, Walgenbach-Brunagel G, von Ruecker A, et al: Analysis of serum microRNAs (miR-26a-2*, miR-191, miR-337-3p and miR-378) as potential biomarkers in renal cell carcinoma. Cancer Epidemiol 36: 391-394, 2012.

37. Wulfken LM, Moritz R, Ohlmann C, Holdenrieder S, Jung V, Becker F, Herrmann E, Walgenbach-Brunagel G, von Ruecker A, Muller SC, et al: MicroRNAs in renal cell carcinoma: Diagnostic implications of serum miR-1233 levels. PLoS One 6: e25787, 2011.

38. Fendler A, Stephan C, Yousef GM, Kristiansen G and Jung K: The translational potential of microRNAs as biofluid markers of urological tumours. Nat Rev Urol 13: 734-752, 2016.

39. White MC, Holman DM, Boehm JE, Peipins LA, Grossman M and Henley SJ: Age and cancer risk: A potentially modifiable relationship. Am J Prev Med 46 (3 Suppl 1): S7-S15, 2014.

40. Miah S, Dudziec E, Drayton RM, Zlotta AR, Morgan SL, Rosario DJ, Hamdy FC and Catto JW: An evaluation of urinary microRNA reveals a high sensitivity for bladder cancer. $\mathrm{Br}$ J Cancer 107: 123-128, 2012.

41. Jiang X, Du L, Wang L, Li J, Liu Y, Zheng G, Qu A, Zhang X, Pan $\mathrm{H}$, Yang $\mathrm{Y}$ and Wang C: Serum microRNA expression signatures identified from genome-wide microRNA profiling serve as novel noninvasive biomarkers for diagnosis and recurrence of bladder cancer. Int J Cancer 136: 854-862, 2015.

42. Binderup HG, Madsen JS, Heegaard NHH, Houlind K Andersen RF and Brasen CL: Quantification of microRNA levels in plasma-Impact of preanalytical and analytical conditions. PLoS One 13: e0201069, 2018

43. Kanaan Z, Roberts H, Eichenberger MR, Billeter A, Ocheretner G, Pan J, Rai SN, Jorden J, Williford A and Galandiuk S: A plasma microRNA panel for detection of colorectal adenomas: A step toward more precise screening for colorectal cancer. Ann Surg 258: 400-408, 2013.

44. Chen Y, Chen J, Liu Y, Li S and Huang P: Plasma miR-15b-5p, miR-338-5p, and miR-764 as biomarkers for hepatocellular carcinoma. Med Sci Monit 21: 1864-1871, 2015.

45. Fogli S, Polini B, Carpi S, Pardini B, Naccarati A, Dubbini N, Lanza M, Breschi MC, Romanini A and Nieri P: Identification of plasma microRNAs as new potential biomarkers with high diagnostic power in human cutaneous melanoma. Tumour Biol 39: 1010428317701646, 2017.

46. Wang F, Zu Y, Zhu S, Yang Y, Huang W, Xie H and Li G: Long noncoding RNA MAGI2-AS3 regulates CCDC19 expression by sponging miR-15b-5p and suppresses bladder cancer progression. Biochem Biophys Res Commun 507: 231-235, 2018.

47. Zhao C,Li Y, Chen G, Wang F, Shen Zand Zhou R: Overexpression of miR-15b-5p promotes gastric cancer metastasis by regulating PAQR3. Oncol Rep 38: 352-358, 2017.

48. Summerer I, Niyazi M, Unger K, Pitea A, Zangen V, Hess J, Atkinson MJ, Belka C, Moertl S and Zitzelsberger H: Changes in circulating microRNAs after radiochemotherapy in head and neck cancer patients. Radiat Oncol 8: 296, 2013.

49. Yu M, Luo Y, Cong Z, Mu Y, Qiu Y and Zhong M: MicroRNA-590-5p inhibits intestinal inflammation by targeting YAP. J Crohns Colitis 12: 993-1004, 2018.

50. Dong L, Lin F, Wu W, Liu Y and Huang W: Verteporfin inhibits YAP-induced bladder cancer cell growth and invasion via Hippo signaling pathway. Int J Med Sci 15: 645-652, 2018.
51. Liu JY, Li YH, Lin HX, Liao YJ, Mai SJ, Liu ZW, Zhang ZL, Jiang LJ, Zhang JX, Kung HF, et al: Overexpression of YAP 1 contributes to progressive features and poor prognosis of human urothelial carcinoma of the bladder. BMC Cancer 13: 349, 2013.

52. van Kessel KE, Van Neste L, Lurkin I, Zwarthoff EC and Van Criekinge W: Evaluation of an epigenetic profile for the detection of bladder cancer in patients with hematuria. J Urol 195: 601-607, 2016.

53. Zhang J, Zhou Y, Huang T, Wu F, Pan Y, Dong Y, Wang Y, Chan AK, Liu L, Kwan JS, et al: FGF18, a prominent player in FGF signaling, promotes gastric tumorigenesis through autocrine manner and is negatively regulated by miR-590-5p. Oncogene 38: 33-46, 2019.

54. Marzi MJ, Ghini F, Cerruti B, de Pretis S, Bonetti P, Giacomelli C, Gorski MM, Kress T, Pelizzola M, Muller H, et al: Degradation dynamics of microRNAs revealed by a novel pulse-chase approach. Genome Res 26: 554-565, 2016.

55. Gantier MP, McCoy CE, Rusinova I, Saulep D, Wang D, Xu D, Irving AT, Behlke MA, Hertzog PJ, Mackay F and Williams BR: Analysis of microRNA turnover in mammalian cells following Dicer1 ablation. Nucleic Acids Res 39: 5692-5703, 2011

56. Guo Y, Liu J, Elfenbein SJ, Ma Y, Zhong M, Qiu C, Ding Y and $\mathrm{Lu}$ J: Characterization of the mammalian miRNA turnover landscape. Nucleic Acids Res 43: 2326-2341, 2015.

57. Juracek J, Peltanova B, Dolezel J, Fedorko M, Pacik D, Radova L, Vesela P, Svoboda M, Slaby O and Stanik M: Genome-wide identification of urinary cell-free microRNAs for non-invasive detection of bladder cancer. J Cell Mol Med 22: 2033-2038, 2018.

58. Zhang X, Zhang Y, Liu X, Fang A, Wang J, Yang Y, Wang L, Du L and Wang C: Direct quantitative detection for cell-free miR-155 in urine: A potential role in diagnosis and prognosis for non-muscle invasive bladder cancer. Oncotarget 7: 3255-3266, 2016.

59. Du L, Jiang X, Duan W, Wang R, Wang L, Zheng G, Yan K, Wang L, Li J, Zhang X, et al: Cell-free microRNA expression signatures in urine serve as novel noninvasive biomarkers for diagnosis and recurrence prediction of bladder cancer. Oncotarget 8: 40832-40842, 2017.

60. Tsujiura M, Ichikawa D, Komatsu S, Shiozaki A, Takeshita H, Kosuga T, Konishi H, Morimura R, Deguchi K, Fujiwara H, et al: Circulating microRNAs in plasma of patients with gastric cancers. Br J Cancer 102: 1174-1179, 2010.

61. Le HB, Zhu WY, Chen DD, He JY, Huang YY, Liu XG and Zhang YK: Evaluation of dynamic change of serum miR-21 and miR-24 in pre- and post-operative lung carcinoma patients. Med Oncol 29: 3190-3197, 2012 .

62. Heneghan HM, Miller N, Lowery AJ, Sweeney KJ, Newell J and Kerin MJ: Circulating microRNAs as novel minimally invasive biomarkers for breast cancer. Ann Surg 251: 499-505, 2010.

63. Aushev VN, Zborovskaya IB, Laktionov KK, Girard N, Cros MP, Herceg Z and Krutovskikh V: Comparisons of microRNA patterns in plasma before and after tumor removal reveal new biomarkers of lung squamous cell carcinoma. PLoS One 8: e78649, 2013.

64. Leidinger P, Keller A, Backes C, Huwer H and Meese E: MicroRNA expression changes after lung cancer resection: A follow-up study. RNA Biol 9: 900-910, 2012.

65. Leidinger P, Galata V, Backes C, Stähler C, Rheinheimer S, Huwer H, Meese E and Keller A: Longitudinal study on circulating miRNAs in patients after lung cancer resection. Oncotarget 6: 16674-16685, 2015.

66. Egidi MG, Cochetti G, Serva MR, Guelfi G, Zampini D, Mechelli L and Mearini E: Circulating microRNAs and kallikreins before and after radical prostatectomy: Are they really prostate cancer markers? Biomed Res Int 2013: 241780, 2013.

67. Keller A, Rounge T, Backes C, Ludwig N, Gislefoss R, Leidinger $\mathrm{P}$, Langseth $\mathrm{H}$ and Meese E: Sources to variability in circulating human miRNA signatures. RNA Biol 14: 1791-1798, 2017.

68. Aarsand AK, Roraas T, Fernandez-Calle P, Ricos C, Diaz-Garzon J, Jonker N, Perich C, Gonzalez-Lao E, Carobene A, Minchinela J, et al: The biological variation data critical appraisal checklist: A standard for evaluating studies on biological variation. Clin Chem 64: 501-514, 2018.

69. Burke HB: Increasing the power of surrogate endpoint biomarkers: The aggregation of predictive factors. J Cell Biochem (Suppl 19): S278-S282, 1994. 